\title{
Interactive Computation and Visualization of Structural Connectomes in Real-Time
}

\author{
Maxime Chamberland ${ }^{1}$, Liam Gray ${ }^{1}$, Maxime Descoteaux ${ }^{2}$ and Derek K. \\ Jones $^{1}$ \\ ${ }^{1}$ CUBRIC, Cardiff University, Cardiff, United Kingdom \\ ${ }^{2}$ SCIL, University of Sherbrooke, Sherbrooke, Canada
}

\begin{abstract}
Structural networks contain high dimensional data that raise huge computational and visualization problems, especially when attempting to characterise them using graph theory. As a result, it can be nonintuitive to grasp the contribution of each edge within a graph, both at a local and global scale. Here, we introduce a new platform that enables tractography-based networks to be explored in a highly interactive real-time fashion. The framework allows one to interactively tune graphrelated parameters on the fly, as opposed to conventional visualization softwares that rely on pre-computed connectivity matrices. From a neurosurgical perspective, the method also provides enhanced understanding regarding the potential removal of a specific node or transection of an edge from the network, allowing surgeons and clinicians to discern the value of each node.
\end{abstract}

\section{Introduction}

The human brain can be viewed as a network2009. This highly specialized network can be conceptualized to as a set of nodes (e.g. gray matter (GM) regions) linked together by edges (e.g. white matter (WM)). Brain networks derived from graph theory analyses are often dense and complex, and thus perceptually challenging to visualize 2013. While thresholding edges can help reduce the complexity of a network, it often leads to high variance in graph metrics (VanWijk et al. 2010, Fornito et al. 2013, Drakesmith et al. 2015). Moreover, false positive in tractography (Cote et al. 2013, Maier-Hein et al. 2016) pollute connectivity matrices and have direct impact on the various graph metrics. To better understand the role of these confounding factors on network topology, we developed a new platform for exploring structural networks in a highly-interactive fashion. The proposed visualization framework: 1) provides real-time insight of various thresholds on graph metrics; and 2) enables a seamless transition between an abstract (graph and edges) and an anatomical (streamlines) representation, allowing one to inspect the underlying architecture of a specific edge. 


\section{Methods}

\subsection{Structural Connectivity}

Diffusion-weighted images of a single-subject were acquired along 64 uniformlydistributed directions at $\mathrm{b}=1000 \mathrm{~s} / \mathrm{mm}^{2}$, using single-shot EPI on a 1.5 Tesla SIEMENS Magnetom $(128 \times 128$ matrix, $2 \mathrm{~mm}$ isotropic resolution, TR/TE 11000/98 ms) and a GRAPPA factor of 2. An anatomical T1-weighted $1 \mathrm{~mm}$ isotropic MPRAGE (TR/TE 6.57/ $2.52 \mathrm{~ms}$ ) image was also acquired for the estimation of partial volume maps (PVE). The diffusion-weighted images were upsampled to the anatomical resolution (1 $\mathrm{mm}$ isotropic). Fiber Orientation Distribution Functions from spherical deconvolution (Tournier et al. 2007) were used for tractography. PVE maps were used in the tracking process to provide a better tracking domain as opposed to fractional anisotropy (FA)-based mask where streamline propagation is often prematurely halted in crossing regions. Probabilistic Particle Filtering Tractography (Girard et al. 2014) was done seeding from the white matter $(\mathrm{WM})$ and grey matter $(\mathrm{GM})$ interface $(1 \times 1 \times$ $1 \mathrm{~mm}^{3}, 2 \mathrm{M}$ seeds). The particle filtering tractography algorithm ensured that streamlines did not terminate prematurely in the WM by the application of a back-tracking rule to allow the tractography algorithm to find alternative pathways. Freesurfer (Fischl et al. 2004) was used to parcellate the brain into 163 labels (Destrieux et al. 2009). Subcortical regions were included to ensure an accurate representation of WM connections throughout the brain (e.g. thalamocortical radiations). The same reasoning was applied to the brain stem and cerebellum regions to ensure the inclusion of the corticospinal/corticocerebellar tracts within the graph. A $3 \mathrm{~mm}$ dilation was used to ensure a robust overlap between streamlines end-points (e.g. GM/WM interface) and anatomical labels (Yeh et al. 2016). Finally, streamlines and brain labels were loaded in the FiberNavigator $^{1}$ (Chamberland et al. 2015).

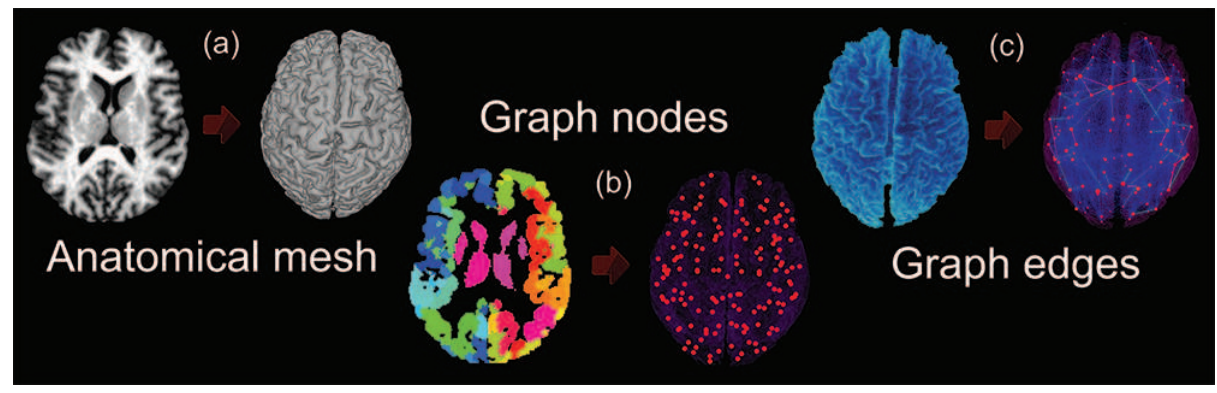

Fig. 1. Graph construction. a) Mesh derived from anatomical T1 image. b) Nodes derived from anatomical labels. c) Edges derived from tractogram.

\footnotetext{
${ }^{1}$ Open source software available at: chamberm.github.io/fibernavigator_single.html
} 


\section{Visualization}

First, an iso-surface is derived from the T1-weighted image for contextual reference (Fig. 1). Next, a spherical node (red) is positioned at the barycenter of each label. A default weighted connectivity matrix $(\mathbf{M})$ is built by normalizing the number of streamlines linking each anatomical region ${ }^{2}$. A transfer function is responsible for mapping values of $\mathbf{M}$ towards edge thickness and opacity. The default view also resizes each node by its degree and a side panel shows a set of global graph metrics (e.g., mean degree, global efficiency). Selecting a node instantly initiates the computation of node-related metrics (e.g. degree, strength, centrality, efficiency). In addition, selecting any 2 nodes immediately reveals the underlying streamlines forming the edge between them. An interactive global threshold (acting on the weights of $\mathbf{M}$ ) is also available, which automatically updates the global and local metrics of the network on the fly, as well as the visualization of the graph. Finally, to reduce visual ambiguity in node selection, nodes are depth sorted and color-graded in real-time according to the current viewpoint. Importantly, although very fast, the new framework is implemented on CPU using $\mathrm{C}++$ and GLSL shaders, can run on a single core computer, and does not require any specific hardware. Experimentations were performed on a laptop with the following specifications: System: Windows 8, Video card: Geforce GT 640M memory 2GB, NVIDIA Driver: 306.97, CPU: Intel(R)Core(TM) i73632QM @ 2,20GHz, 16GB RAM.

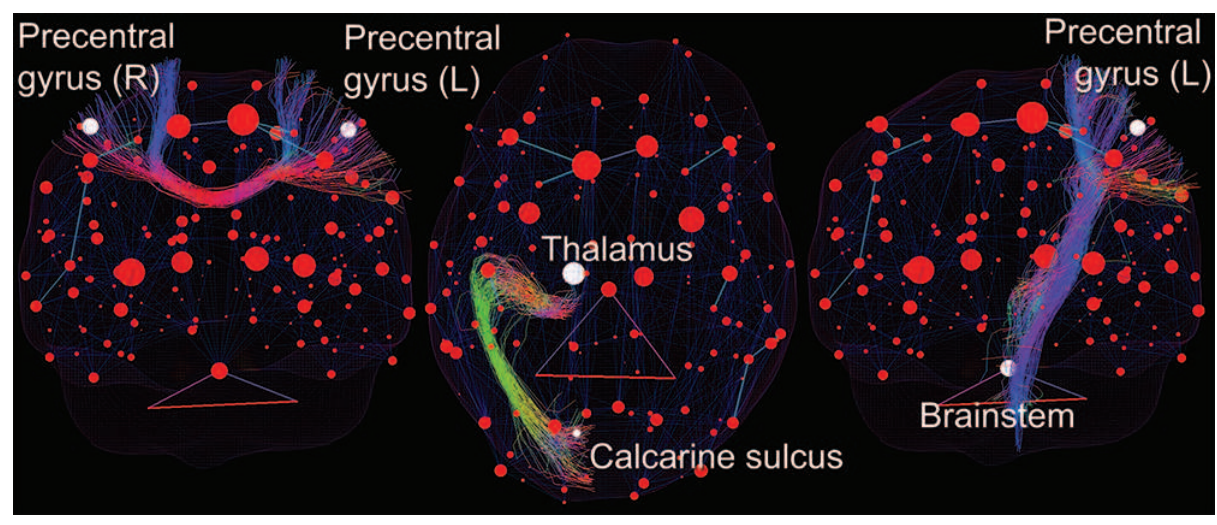

Fig. 2. Bundle selection using node picking (white).

\footnotetext{
${ }^{2}$ Demo available online at: www.youtube.com/watch?v=eZ2JubD25NA
} 


\section{Results}

Underlying streamlines linking 2 nodes are illustrated in Fig. 2. From left to right: corpus callosum (CC), optic radiation (OR) and corticospinal tract (CST). Controversial streamlines forming thick edges in the graph (number of streamlines in this case) are easily identified (e.g. Frontal Aslant Tract (FAT) (Catani et al. 2013)) and can potentially be removed from the network (e.g. $\mathrm{M}_{i j}=0$ ) (Fig. 3).

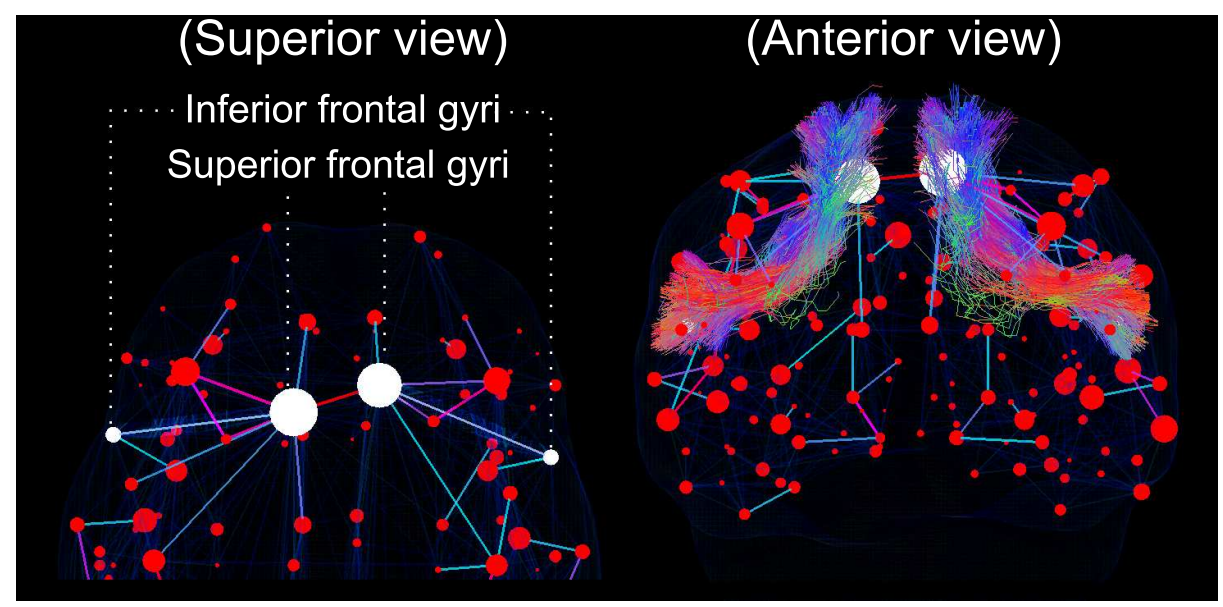

Fig. 3. Frontal Aslant Tract (FAT) (Catani et al. 2013)) rapidly identified by the selection of 2 nodes.

Fig. 4 shows two versions of the whole-brain network (i.e. unthresholded vs thresholded) as well as its associated global and nodal graph metrics (Tables 1, 2). Given a specific node of interest (e.g. pre-central gyrus, Fig. 4 yellow), the user can instantaneously observe variations in the different metrics related to that node by dragging the threshold slider ( $2 \%$ threshold). A 30 frame-persecond (FPS) ratio was maintained during the process. 
Table 1. Real-time global graph metrics

\begin{tabular}{lcc}
\hline Metrics & Default graph & Thresholded graph $(2 \%)$ \\
\hline \# of nodes & 161 & 160 \\
\# of edges & 4632 & 938 \\
Density & 0.36 & 0.07 \\
Mean degree & 62.59 & 12.68 \\
Global efficiency & 0.446 & 0.104 \\
\hline
\end{tabular}

Table 2. Real-time local graph metrics (right pre-central gyrus)

\begin{tabular}{lcc}
\hline Metrics & Default graph & Thresholded graph $(2 \%)$ \\
\hline Degree & 88 & 24 \\
Strength & 3.73 & 3.49 \\
Eigen centrality & 0.165 & 0.161 \\
Closeness centrality & 0.919 & 0.691 \\
Local efficiency & 0.896 & 0.955 \\
\hline
\end{tabular}

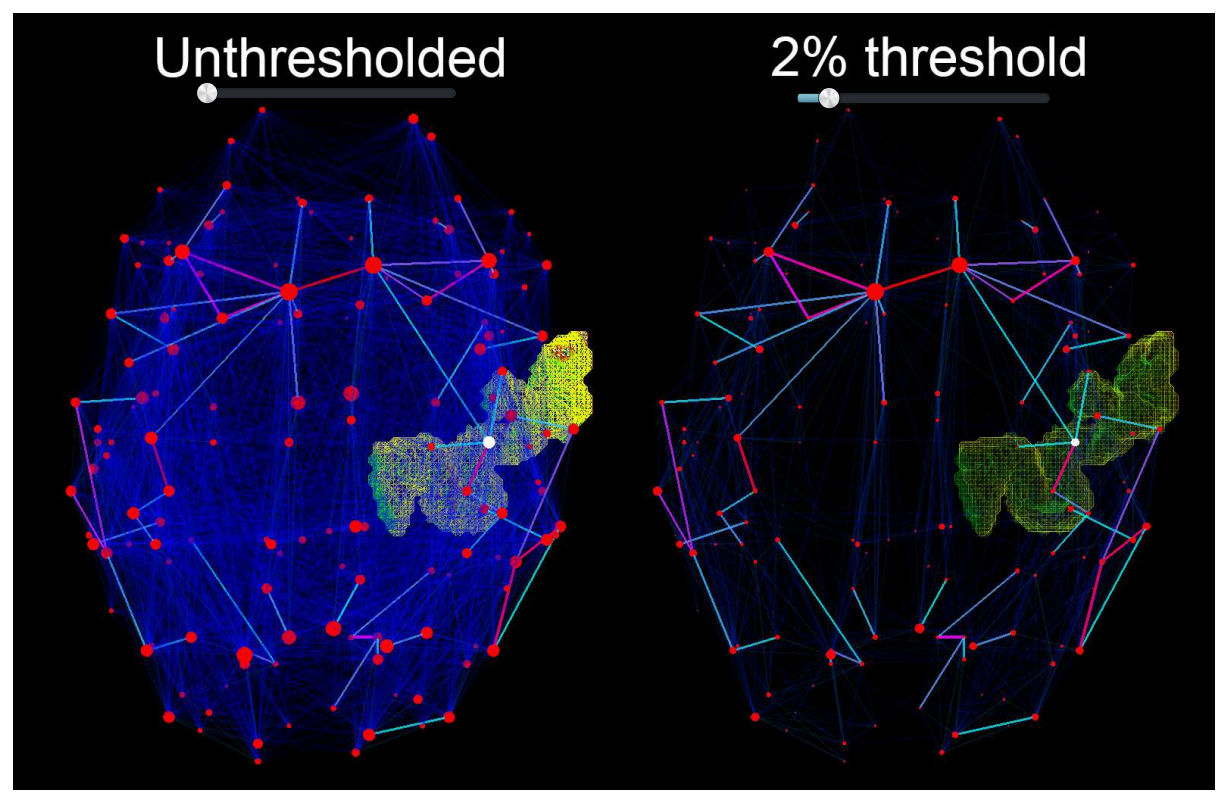

Fig. 4. Threshold graph visualization. Node sizes are recomputed on the fly according to their new strength. Yellow: pre-central gyrus (R). 
Finally, Fig. 5 shows how depth-sorting can help differentiate occipital nodes from frontal nodes. For any viewpoint, a transfer function assigns a color grading to each node based on their $\mathrm{Z}$ position in the scene. In this example, nodes located in the posterior aspect of the brain appear brighter than the ones located in the frontal lobe since the camera is looking at the brain from behind. Finally, a supplementary video illustrating the aforementioned options is also available online at: www.youtube.com/watch?v=eZ2JubD25NA.

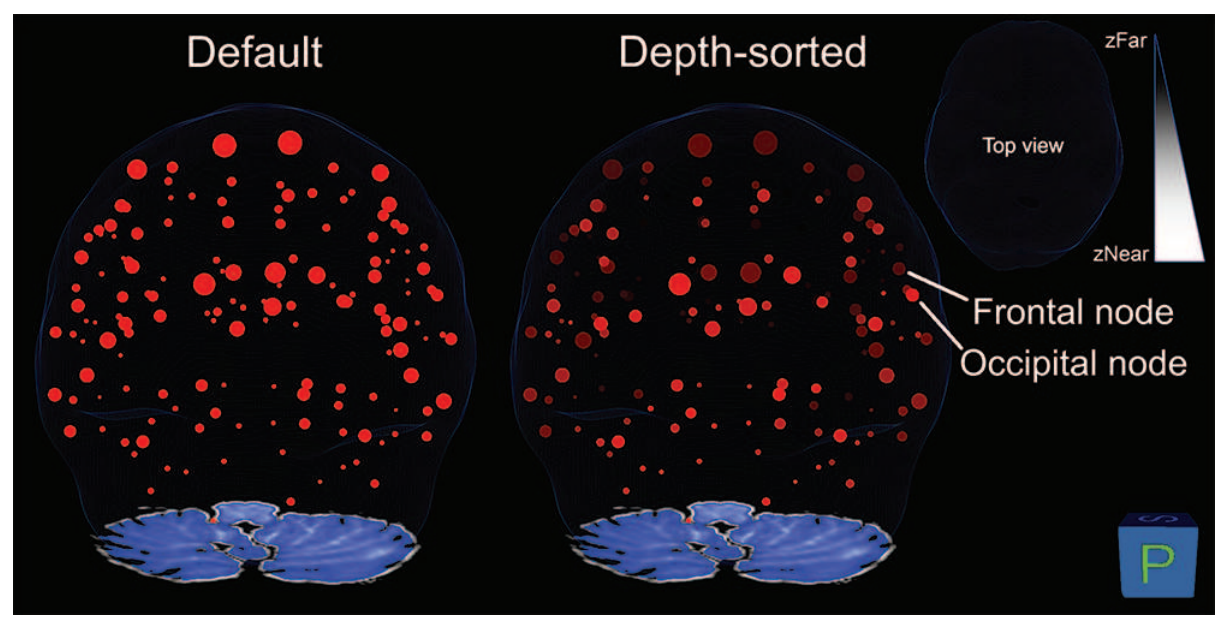

Fig. 5. Depth-sorted nodes provide increase visual cues when compared to default rendering.

\section{Discussion}

To the best of our knowledge, this is the first visualization platform supporting comprehensive exploration of structural connectomics in real-time. The tool allows the user to easily prune undesired edges of the graph (e.g. false-positive streamlines). The mean FPS ratio was above 30 during all steps, indicating no latency. Initial piloting of the tool (by users new to graph theory) revealed the following consensus: hubs and underlying streamlines were easily identifiable by all. Moreover, participants were mostly curious how simple threshold manipulation altered local and global network metrics. After discussing with neurosurgeons, the framework also incorporates various representation of $\mathbf{M}$ by allowing direct manipulation of bundle-specific edge weights (e.g. to simulate de- or remyelination and its effect on the network). The current version also allows users to input a more general connectivity matrix (e.g. derived from other softwares or modalities such as resting-state functional MRI or MEG). In other words, the users are not bound to a specific tractography pipeline to generate the afore- 
mentioned connectivity matrix. Moreover, it is important to specify that any set of brain parcellation can be used here (i.e. varying number of labels).

\section{Conclusion}

With the large variety of metrics and parameters involved in connectomics (e.g. weights of $\mathbf{M}$, threshold techniques (Drakesmith et al. 2015)), the proposed growing visualization framework will also serve as a quality assurance tool for close inspection of data prior to launching massive analyses. From a clinical perspective, the proposed platform will also provide neurosurgeons with a better understanding of the effect of transecting pathways underlying critical hubs, and perhaps physiotherapists insight into the impact of strengthening a given edge on network characteristics. 


\section{$7 \quad$ References}

\section{References}

Bullmore Ed., and Sporns O. Complex brain networks: graph theoretical analysis of structural and functional systems. Nature reviews. Neuroscience 10.3 (2009): 186.

Catani M., Mesulam M. M., Jakobsen E., Malik F., Martersteck A., Wieneke C., Thompson C.K. et al. A novel frontal pathway underlies verbal fluency in primary progressive aphasia. Brain 136, no. 8 (2013): 2619-2628.

Chamberland M., Bernier M., Fortin D., Whittingstall K., and Maxime Descoteaux. $3 \mathrm{D}$ interactive tractography-informed resting-state fMRI connectivity. Frontiers in neuroscience 9 (2015): 275.

Cote, M-A., Girard G., Bor A., Garyfallidis E., Houde J-C., and Maxime Descoteaux. Tractometer: towards validation of tractography pipelines. Medical image analysis 17, no. 7 (2013): 844-857.

Destrieux C., Fischl B., Dale A. M. , and E. Halgren. A sulcal depth-based anatomical parcellation of the cerebral cortex. NeuroImage 47 (2009): S151.

Drakesmith M., Karen Caeyenberghs, A. Dutt, G. Lewis, A. S. David, and Derek K. Jones. Overcoming the effects of false positives and threshold bias in graph theoretical analyses of neuroimaging data. NeuroImage 118 (2015): 313-333.

Fischl B., Van Der Kouwe A., Destrieux C., Halgren E., Sgonne F., Salat D. H., Evelina B. et al. Automatically parcellating the human cerebral cortex. Cerebral cortex 14, no. 1 (2004): 11-22.

Fornito A., Andrew Z., and Michael Breakspear. Graph analysis of the human connectome: promise, progress, and pitfalls. Neuroimage 80 (2013): 426-444.

Girard G., Kevin, W., Deriche R., and Maxime Descoteaux. Towards quantitative connectivity analysis: reducing tractography biases. Neuroimage 98 (2014): 266278.

Maier-Hein K., Neher P., Houde J-C., Cote M-A., Garyfallidis E., Zhong J., Chamberland M. et al. Tractography-based connectomes are dominated by false-positive connections. bioRxiv (2016): 084137.

Margulies, D. S., Bttger, J., Watanabe, A., and Gorgolewski, K. J. Visualizing the human connectome. NeuroImage, 80 (2013), 445-461.

Tournier J-D., Fernando C., and Alan Connelly. Robust determination of the fibre orientation distribution in diffusion MRI: non-negativity constrained super-resolved spherical deconvolution. NeuroImage 35.4 (2007): 1459-1472.

Van Wijk B. CM., Cornelis J. S., and Andreas Daffertshofer. Comparing brain networks of different size and connectivity density using graph theory. PloS one 5, no. 10 (2010): e13701.

Yeh C-H., Smith R., Dhollander T., Calamante F., Connelly A. The Influence of Node Assignment Strategies and Track Termination Criteria on Diffusion MRIBased Structural Connectomics International Symposium on Magnetic Resonance in Medicine (ISMRM), no. 0118 (2016). 